\title{
A 3D Ellipsoidal Volumetric Foot-Ground Contact Model for Forward Dynamics
}

\author{
Peter Brown John McPhee
}

September 18, 2017

\section{Abstract}

Foot-ground contact models are an important part of forward dynamic biomechanic models, particularly those used to model gait, and have many challenges associated with them. Contact models can dramatically increase the complexity of the multibody system equations, especially if the contact surface is relatively large or conforming. Since foot-ground contact has a large potential contact area, creating a computationally efficient model is challenging. This is particularly problematic in predictive simulations, which may determine optimal performance by running a model simulation thousands of times. An ideal contact model must find a balance between accuracy for large, conforming surfaces, and computational efficiency.

Volumetric contact modelling is explored as a computationally efficient model for footground contact. Previous foot models have used volumetric contact before, but were limited to 2D motion and approximated the surfaces as spheres or 2D shapes. The model presented here improves on current work by using ellipsoid contact geometry and considering 3D motion and geometry. A gait experiment was used to parametrise and validate the model. The model ran over 100 times faster than real-time (in an inverse simulation at $128 \mathrm{fps}$ ) and matched experimental normal force and centre of pressure location with less than $7 \%$ root-mean-square error.

In most gait studies, only the net reaction forces, centre of pressure, and body motions are recorded and used to identify parameters. In this study, contact pressure was also recorded and used as a part of the identification, which was found to increase parameter optimisation time from $10 \mathrm{~s}$ to $164 \mathrm{~s}$ (due to the additional time needed to calculate the pressure distribution) but helped the results converge to a more realistic model. The model matched experimental pressures with 33-45\% root-mean-square error, though some of this was due to measurement errors.

The same parametrisation was done with friction included in the foot model. It was determined that the velocity-based friction model that was used was inappropriate for use in an inverse-dynamics simulation. Attempting to optimise the model to match experimental friction resulted in a poor match for friction forces, inaccurate values for the coefficient of friction, and a poorer match to the experimental normal force. 


\section{Introduction}

In forward dynamics simulation, or predictive simulation, a set of inputs (such as muscle forces) is used to drive a human model to predict human motion. A forward dynamics model can be used in an optimisation routine with a physiological cost function to predict human motion in untested situations or to predict human movement optimised for certain criteria. Optimisation requires many simulations to converge to a solution (often thousands) so a forward dynamics model must be computationally efficient as well as accurate for use in predictive simulations. Predictive simulation is currently an active area of research, and can be used to determine possible balance control and human energy optimisation methods [1], [2], to predict consequences of therapy or surgery [3], [4], and to optimise athlete performance or equipment [5].

One important aspect of biomechanical simulations are the contact models, especially foot-ground contact. An accurate contact model is required to calculate ground reaction forces and pressures, which is necessary for accurate replication of human motion. Also, contact models can predict tissue loads due to impact and contact, which may be used in human movement optimisation to improve prosthetic longevity [3] or reduce tissue loads $[6]$.

Contact models introduce many challenges; contact forces are often nonlinear, discontinuous, and result in stiff system equations. The foot plantar surface is compliant and has a large area of contact on the ground. Additionally, the ground is often hard to model since it is not always flat, especially in outdoor settings. It is challenging to develop a model of sufficient accuracy that is still efficient enough to use in an optimisation.

The focus of this research is on contact models that can be used in forward dynamics simulations and to predict ground reaction forces and pressures throughout contact. This means that models for inverse dynamics simulations [7]-[9] are not useful in this context, since they can only predict forces from experimental motion. Also, although impulsive models [10], [11] yield practically the same motion as continuous contact models, they provide little information on contact forces, which is less useful for certain studies. A continuous contact model can be used in a forward dynamic simulation and calculates the contact forces throughout impact and contact. Thus, this research will focus on compliant, continuous contact models.

Of continuous contact models, detailed and accurate contact models that can account for conforming contacts tend to be computationally expensive, making them poorly suited for optimisations [6], [12]. Simpler contact models are often based on point-contact models (such as the Hunt-Crossley model [13]) which are computationally efficient, but are only accurate for small contact patches (not conforming surfaces). This issue can be partially alleviated by using a large number of point contacts over the contact surface [1], [14][16]. Alternatively, point contact models can be modified by using various geometries to represent the contact surface (such as spheres, disks, or superellipsoids) [15], [17], [18]; this geometry determines the central or most important point of contact at which the contact forces are calculated. In cases like this, it is more computationally efficient to use analytical shapes, such as spheres or superellipsoids, than meshes to represent the contact surfaces [19]. A balance is needed between a simplified model that will be computationally efficient, and an accurate model. 
Volumetric contact shows potential as an efficient continuous foot-ground contact model. Volumetric contact, first proposed by Gonthier et al. for contact in robotic manipulators [20], is based on the elastic foundation model, which models the surface as a continuous collection of springs [20], as shown in Figure 1.
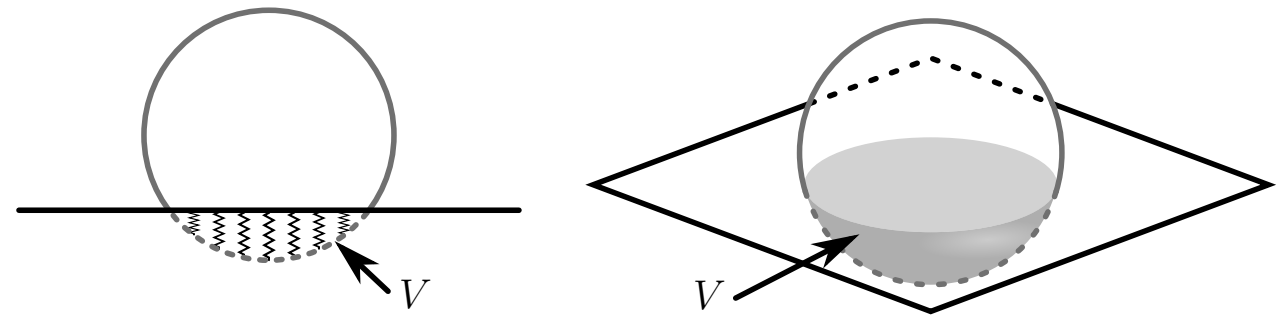

Figure 1: Volumetric contact surface interaction (sphere-on-plane)

Since volumetric contact considers the pressure developed across the whole contact surface, it is more accurate than point contact models for complex and conforming geometries [21]. This also means that the pressure across the contact surface can be calculated naturally with volumetric contact, unlike point contact models. The contact forces can be found as an integral of the stresses developed across the contact area. If the contact geometry is represented as simplified shapes - which is generally done for volumetric contact - the distributed forces over the contact surface can be integrated to obtain analytical equations for the equivalent forces and torques at a resultant point. Volumetric contact includes a damping term which can capture some of the viscoelasticity of the footpad, but the standard form assumes a linear stiffness, and may lose some accuracy when modelling materials with non-linear stiffness. In addition to the normal force, volumetric contact defines equations for rolling resistance, tangential friction, and spinning friction. This results in a set of equations much more efficient than discrete elastic foundation models, and not significantly more complex than point contact models.

For volumetric contact, if the surface stiffness is linear, integration across the contact surface reveals that the normal force is directly proportional to the volume of penetration of the two surfaces ( $V$ in Figure 1$)$ :

$$
F_{n}=k_{V} V\left(1+a_{V} v_{c n}\right)
$$

where $V$ is the volume of penetration, $v_{c n}$ is the relative velocity of the two surfaces in the normal direction at the volumetric centroid, $k_{V}$ is the volumetric stiffness, and $a_{V}$ is the damping constant. This equation holds for contact between any two objects, where $V$ is the volume shared by both objects.

Similarly, the centre of pressure (COP) is at the centroid of this volume of penetration, and all other contact forces (rolling resistance and friction) can be related to the volume of penetration and its properties [21], [22]. The equations for the other contact forces are not given here, but a general derivation is in Gonthier's thesis [22].

Volumetric contact was first used in foot-ground contact by Millard et al. [23], who used a 2D two-segment foot model with three volumetric sphere-plane contact pairs. This model was further developed by Sharif Shourijeh et al. who also proposed a hyper-volumetric model [24], [25]. Mouzo et al. used volumetric contact in a 2D foot model with a polynomial 
representing the contact surface [26]. Volumetric contact has yet to be used in a 3D foot model.

To expand on previous work, the plantar surface was approximated as ellipsoids, which can match complex geometry more closely than spheres can. The volumetric contact equations for an ellipsoid contacting a plane were derived in [27], [28]. These equations included normal force damping, rolling resistance, lateral friction, and spinning friction.

The primary contribution of this work is a computationally efficient, 3D contact model of the foot, utilising volumetric contact. To the authors' best knowledge, this is the first use of volumetric contact in a 3D foot model. Additionally, this is the first study in which, to the authors' best knowledge, that experimental pressure measurements were used to parametrise a foot model; similar studies have only measured the net forces and moments (or centre of pressure location) [16], [29].

\section{Foot Model}

The foot-ground contact model was constructed in MapleSim. The foot model was made of two rigid segments: the foot segment (including the heel and ball of the foot) and the toe segment. The two segments were connected by a revolute joint representing the toe joint. The contact geometry was represented using three ellipsoids contacting the ground. Two ellipsoids were used to represent the foot segment (one for the heel and one for the ball of the foot), and one ellipsoid to represent the toe segment (as shown in Figure 2). These location were chosen based on measurements taken using a pressure mat (discussed further in Section 3.2.3). Detailed frame definitions and the toe axis location is given in Section 3.1.1. For the initial model, to reduce complexity, all ellipsoid contacts were assumed to have the same volumetric stiffness and damping. This resulted in a model with 29 parameters (listed in Table 1).

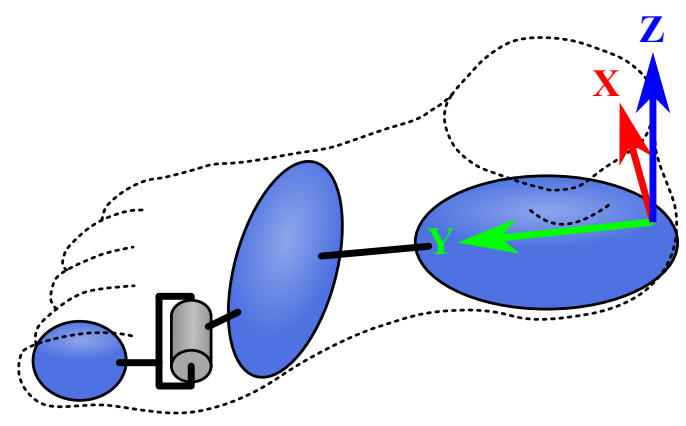

Figure 2: Foot model

The MapleSim model was exported as C code and compiled in Matlab (R2016a. MathWorks, Natick, MA, USA) for use in an inverse dynamics optimisation. This inverse model ran over 100 times faster than real-time (running at 128 frames per second, to match the experimental data collection rate). However, this does not include calculating the pressure across the contact area, which was used in some of the optimisations. When the simulation was also used to calculate the pressure distribution (at 15 timeframes, on a 39x16 grid), the simulation ran about 15 times faster than real-time. 
Table 1: Foot-ground contact model parameters to identify

\begin{tabular}{l|c|c|c} 
Scope & Parameter(s) & Description & Units \\
\hline & $k_{V}$ & Volumetric stiffness & $\mathrm{N} / \mathrm{m}^{3}$ \\
Global & $a_{V}$ & Volumetric damping coefficient & $\mathrm{s} / \mathrm{m}$ \\
\hline Ellipsoid geometry & $a, b, c$ & Dimensions & $\mathrm{m}$ \\
(for each ellipsoid) & $r_{x}, r_{y}, r_{z}$ & Position & $\mathrm{m}$ \\
& $\alpha, \beta, \gamma$ & $1-2-3$ body-fixed Euler angles & $\mathrm{rad}$
\end{tabular}

${ }^{1}$ Parameters $r_{x}, r_{y}$, and $r_{z}$ specify the location of the contact ellipsoid centroid with respect to the foot or toe frame (defined in Section 3.1.1); $\alpha, \beta$, and $\gamma$ specify the rotation of the local frame for the ellipsoid; and $a, b$, and $c$ specify the ellipsoid radii in the local $x, y$, and $z$-axis, respectively.

\section{Experimental Parametrisation Method}

A gait experiment was used to parametrise and validate the foot model. Gait was chosen as it is one of the primary desired applications of the model. All experiments used only one subject and one type of movement (gait), so the model developed in this paper should be considered a subject specific model. Using a single subject may limit the use of this model, but may be beneficial for accuracy, since subject-specific contact models tend to improve accuracy [30], [31], especially if intersegmental foot loading would be of interest due to the intersubject variability in foot morphology [32].

\subsection{Experimental Protocol}

For the gait experiment, one participant (male, $24 \mathrm{yr}, 185 \mathrm{~cm}, 70 \mathrm{~kg}$ ) was asked to walk naturally while the left foot and lower leg motion, ground reaction forces, and foot-ground contact pressures were recorded as detailed below. The subject's natural gait cadence was measured as 92 steps/minute, and five trials were conducted at this cadence. Five more trials were run at a higher cadence (112 steps/minute) and five more at a lower cadence (72 steps/minute). The gait cadence was enforced using a metronome: a metronome was played at the desired cadence and the subject was instructed to step in time with the tones (several trial runs were allowed for the subject to become familiar with the cadence).

Motion was recorded using Optotrak active markers (Certus. NDI, Waterloo, ON, Canada). Motion data was collected at $128 \mathrm{~Hz}$. One marker cluster was placed on the foot, one marker cluster on the shank, one marker on the first toe, and one on the fourth and fifth toes (which were taped together in order to secure the marker) as shown in Figure 3 .

During a standing calibration trial, the following markers were placed and recorded relative to the foot cluster: the lateral and medial malleoli, the heel (posterior protrusion of the calcaneus), the distal heads of metatarsal 1, 3, and 5 , and the 1st, 3rd, and 5th toes. These additional markers were used to locate the toe joint and align the test frame axis.

Ground reaction forces and pressures were measured using an AMTI force plate (OR67. Watertown, MA, USA) and a Tekscan pressure mat (Medical Sensor 3005E for F-Scan system. South Boston, MA, USA) placed on top of the force plate. Force and moment 


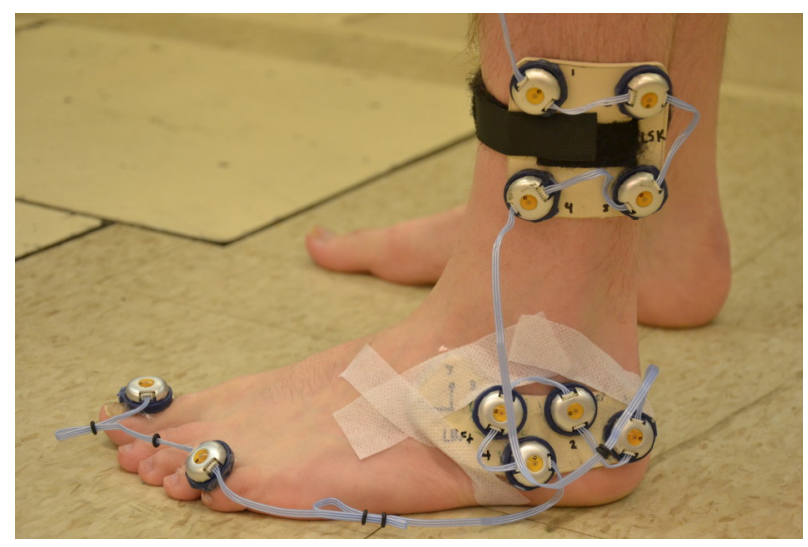

Figure 3: Marker placement on the foot

measurements were collected at $1024 \mathrm{~Hz}$ and pressure measurements at only $64 \mathrm{~Hz}$. The force and moment measures were later down-sampled to $128 \mathrm{~Hz}$ during processing and model optimisation to match the sampling rate of the motion measurements. The force plate and pressure mat locations were measured relative to the Optotrak frame of reference. This allowed the foot position (measured by the motion capture) to be compared with centre of pressure (COP) (measured by the force plate and pressure mat).

It was noted that the pressure mat had a slow response to applied force and measured a total force much lower than the force plate for most of the gait. To partially solve this, the measurements from the pressure mat were scaled such that the total force was equal to that measured by the force plate and less weight was given to the pressure measurements in the optimisation.

To reduce noise, the motion data was filtered with a low-pass filter designed and implemented in Matlab (a dual-pass Butterworth low-pass filter, with the passband at $5 \mathrm{~Hz}$ and stopband at $20 \mathrm{~Hz}$ with 10 times attenuation).

\subsubsection{Frame definitions}

The global frame was set so that the $Z$-axis pointed vertically up, the $Y$-axis pointed in the direction of movement (anterior), and the $X$-axis pointed to the right (medial, for the left foot). The foot frame origin was placed at the heel of the foot with the $y_{\text {foot }}$-axis passing though the 3rd metatarsal and the 1st metatarsal located in the $x_{\text {foot }}-y_{\text {foot }}$ plane (Figure 4).

The toe frame was determined using the metatarsal markers and toe marker, with the

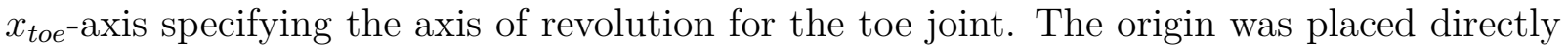
below the 1 st metatarsal with the $x_{t o e}$-axis parallel to the ground and passing under the

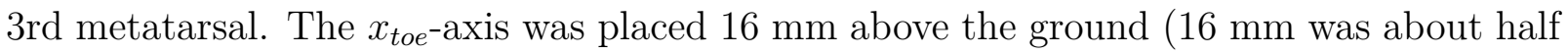
the height of the 3 rd metatarsal). The frame was rotated about the $x_{t o}$-axis so that the toe marker (Toe1) was in the $x_{t o e^{-}} y_{\text {toe }}$ plane. 

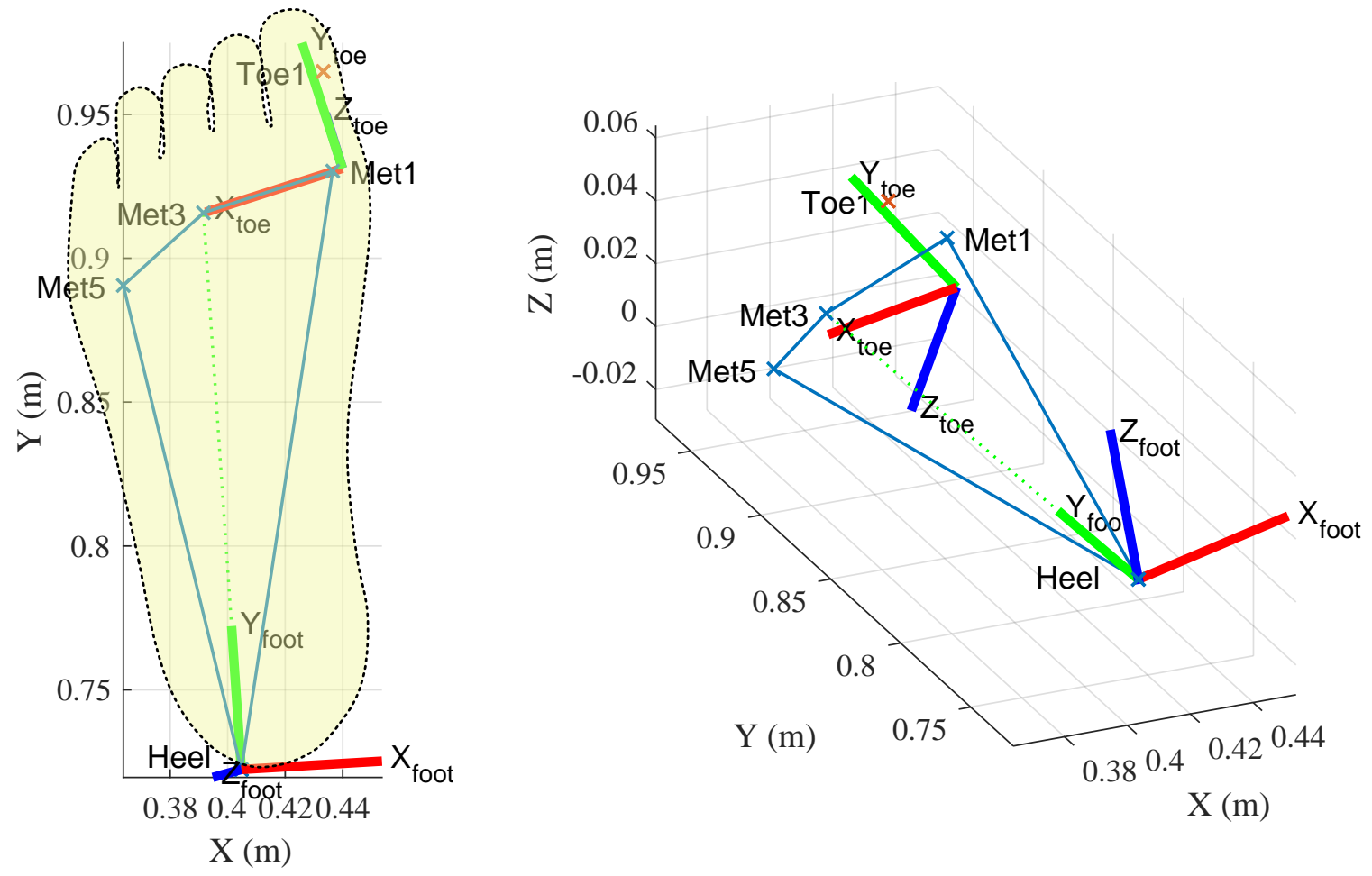

Figure 4: Foot and toe frame conventions (shown in global lab frame)

\subsubsection{Toe rotation and rigid body assumption}

The original intent was to determine the toe joint rotation from the experimental data, but this proved unreliable. When the two-body foot model was fitted to the experimental data, physiologically impossible toe motion was observed - presumably due to errors in determining the toe axis location during movement. These errors were likely caused by the rigid body assumption for the foot and the marker placement. Only one marker cluster was used to determine the foot location, and this was placed near the proximal end of the foot (Figure 3). Internal movement of the foot (i.e. relative movement of the bones that make up the foot) would not have been captured by this marker cluster, so any internal movement would result in errors which were largest near the distal end of the foot (i.e. the toe axis). For the purposes of model simplification, the rigid body (foot) assumption was kept.

Due to the errors noted in the experimentally determined toe movement, the rotation was not calculated from the experiment. For the inverse dynamics study (described later) this rotation was determined purely by optimisation.

\subsection{Parameter Optimisation Method}

To test the model, the foot was subject to the same motion recorded in the gait experiment (in an inverse dynamic simulation) and the simulated ground reaction forces and pressure were compared to the experimental results. 


\subsubsection{Toe rotation and motion correction}

Since the toe rotation could not be determined experimentally, the toe rotation was determined by optimisation. The rotation angle versus time graph was represented by a cubic spline, and the spline data points were included as parameters in the optimisation

To reduce the effect of errors introduced by the rigid body assumption and skin movement, the optimiser was also allowed to adjust the foot motion and orientation. This correction was also specified as a cubic spline, limited to $\pm 2 \mathrm{~mm}$ (for translation in each of the coordinate axes) and $\pm 0.05 \mathrm{rad}$ (for each of the three rotations).

As mentioned previously, there were some errors in the calculation of the toe motion; this error was largest near toe-off. One effect noted during the inverse dynamics simulation was that the simulated toe moved laterally along the ground just before toe-off. This movement was not present physically, but was introduced due to an apparent rotation of the foot. To allow the motion adjustment to correct this error, the lower limit for the $z$ rotation correction was adjusted to $-0.3 \mathrm{rad}$.

The toe rotation and motion correction splines were parametrised by 10 data points evenly spaced over the time of contact. This introduced another 70 parameters (for 3 position correction splines, 3 rotation correction splines, and 1 toe rotation spline) for a total of 99 parameters (including the 29 for the contact model).

\subsubsection{Cost function}

The optimisation cost function was defined as the mean-square difference between the simulation and experimental results for the normal force, COP location, and pressure.

$$
\begin{aligned}
C= & \frac{w_{\text {normal }} s_{\text {normal }}}{N} \sum\left(F_{z \text { exp }}-F_{z \text { sim }}\right)^{2}+ \\
& \frac{w_{\text {cop }} s_{\text {cop }}}{N} \sum\left(\left(x_{\text {cop exp }}-x_{\text {cop sim }}\right)^{2}+\left(y_{\text {cop exp }}-y_{\text {cop sim }}\right)^{2}\right)+ \\
& \frac{w_{\text {pressure }} s_{\text {pressure }}}{N} \sum\left[A_{\text {sensor }} \sum_{x} \sum_{y}\left|p_{\text {xy exp }}-p_{x y \text { sim }}\right|\right]^{2}
\end{aligned}
$$

where $F_{z}$ is the normal force, $x_{\text {cop }}$ and $y_{\text {cop }}$ are the coordinates of the COP, $p_{x y}$ is the pressure at point $(x, y)$ on the pressure sensor, $A_{\text {sensor }}$ is the area of a single sensor on the pressure mat, $N$ is the number of data points, $s$ is a normalisation factor for each term, and $w$ is a non-dimensional weight for each term (for the optimisation). Since multiple units were used in the cost function, the normalisation factors $\left(s_{i}\right)$ were used to obtain scaled, non-dimensional values for each term (the factors are defined in Table 2). The pressure difference is converted into total force difference (by multiplying the pressure measured at each sensor by the area of the sensor), and scaled by the same factor as the normal force term.

The two tangential moments $\left(m_{x}\right.$ and $\left.m_{y}\right)$ were not included in the optimisation since they are a function of the COP and normal force which are already included in the optimisation. The lateral forces and vertical moment were not included since they are functions of friction.

Most other similar studies in the literature have only used net force and COP location to optimise the foot model [16], [29]. In this study, pressure distribution was also used in 
Table 2: Optimisation function normalisation factors

\begin{tabular}{c|c|c}
$s_{i}=\frac{1}{M_{i}^{2}}$ & $M_{i}$ & Description \\
\hline \hline $\begin{array}{c}s_{\text {normal }} \\
s_{\text {pressure }}\end{array}$ & $688 \mathrm{~N}$ & subject weight \\
\hline$s_{\text {cop }}$ & $20 \mathrm{~cm}$ & COP movement distance
\end{tabular}

the optimisation, which is expected to improve the model fidelity. However, calculating the pressure distribution was very computationally expensive, compared to the simulation, so the pressure distribution was only calculated at 15 time-frames for each simulation. The weights for the optimisation function were all set to 1, except for the pressure term ( $\left.w_{\text {pressure }}\right)$ which was set to 0.1 due to the inaccuracies of the pressure measurements.

\subsubsection{Optimisation function}

Optimisation was done using a least-squares solver in MATLAB (lsqnonlin) which uses a trust region reflective algorithm. Optimisation was done on a quad-core desktop (Intel@) Core $^{\mathrm{TM}}$ i7-3770 CPU @ 3.40GHz).

This optimisation required a good initial guess to converge to a reasonable solution. The initial model parameters were adjusted manually such that the three ellipsoids of the heel, ball, and toe matched the locations obtained from the experimental pressure mat measurements. The initial volumetric stiffness was chosen such that the depth of penetration under full-body weight was on the order of $1 \mathrm{~cm}$, and a modest damping factor was chosen. The initial guess used is presented in Table 3.

Table 3: Contact model parameters for initial guess

\begin{tabular}{c|c|c} 
& Parameter(s) & Value \\
\hline \multirow{2}{*}{ Global } & $k_{V}$ & $1 \times 10^{7} \mathrm{~N} / \mathrm{m}^{3}$ \\
& $a_{V}$ & $-1 \mathrm{~s} / \mathrm{m}$ \\
\hline \multirow{3}{*}{ Heel } & $a, b, c$ & $30,50,30 \mathrm{~mm}$ \\
& $r_{x}, r_{y}, r_{z}$ & $0,30,-15 \mathrm{~mm}$ \\
& $\alpha, \beta, \gamma$ & $0,0.15,0 \mathrm{rad}$ \\
\hline \multirow{3}{*}{ Ball } & $a, b, c$ & $60,30,30 \mathrm{~mm}$ \\
& $r_{x}, r_{y}, r_{z}$ & $10,170,-13 \mathrm{~mm}$ \\
& $\alpha, \beta, \gamma$ & $0,0.2,0.5 \mathrm{rad}$ \\
\hline \multirow{3}{*}{ Toe } & $a, b, c$ & $20,20,20 \mathrm{~mm}$ \\
& $r_{x}, r_{y}, r_{z}$ & $0,15,-13 \mathrm{~mm}$ \\
& $\alpha, \beta, \gamma$ & $0,0,0 \mathrm{rad}$
\end{tabular}

During initial tests, the optimisation appeared to only converge to a local minimum. To find a better solution, the initial values were randomly adjusted up to $\pm 10 \%$ several times to find different solutions. Ten different adjustments to the initial conditions in Table 3 were used as initial guesses for the optimisation and the best of the solutions was chosen as the optimal solution. 
Some other optimisation methods, such as a simulated annealing algorithm, inherently include some randomness and may remove the need for randomly adjusting the initial conditions. Other methods were not tested in this work.

The contact model parameters were determined using one gait trial at natural speed using the optimisation method described above (to calibrate the model). The contact model obtained from the calibration was validated by simulating the other gait trials (at the same cadence and the higher and lower cadences). For the other trials, the toe movement and motion correction needed to be determined by optimisation, but the contact model parameters (the 29 parameters in Table 1) were kept the same.

\section{Results}

\subsection{Calibration results}

The optimal parameters are shown in Table 4, the model geometry is shown in Figure 5, and the simulation results are shown in Figure 6. The optimised toe actuation is shown in Figure 7. Optimisation took, on average, $164 \mathrm{~s}$. The RMS (root-mean-square) errors for normal force, COP position, and pressure were $3.4 \%, 3.2 \%$, and $33.9 \%$, respectively.

Table 4: Optimised contact model parameters

\begin{tabular}{c|c|c} 
& Parameter(s) & Value \\
\hline \multirow{2}{*}{ Global } & $k_{V}$ & $1.60 \times 10^{7} \mathrm{~N} / \mathrm{m}^{3}$ \\
& $a_{V}$ & $-0.565 \mathrm{~s} / \mathrm{m}$ \\
\hline \multirow{3}{*}{ Heel } & $a, b, c$ & $35.4,54.0,22.6 \mathrm{~mm}$ \\
& $r_{x}, r_{y}, r_{z}$ & $3.7,47.9,0.4 \mathrm{~mm}$ \\
& $\alpha, \beta, \gamma$ & $0.329,0.201,0.101 \mathrm{rad}$ \\
\hline \multirow{3}{*}{ Ball } & $a, b, c$ & $63.2,33.8,24.0 \mathrm{~mm}$ \\
& $r_{x}, r_{y}, r_{z}$ & $20,167,-0.6 \mathrm{~mm}$ \\
& $\alpha, \beta, \gamma$ & $-0.235,0.191,0.528 \mathrm{rad}$ \\
\hline \multirow{3}{*}{ Toe } & $a, b, c$ & $18.2,18.6,13.2 \mathrm{~mm}$ \\
& $r_{x}, r_{y}, r_{z}$ & $11,10,-19 \mathrm{~mm}$ \\
& $\alpha, \beta, \gamma$ & $0.339,1.23,0.454 \mathrm{rad}$
\end{tabular}

The simulated normal force and the COP were similar to the experimental results $(3.4 \%$ and $3.2 \%$ RMS error, respectively). The shape of the pressure profile during gait (only the maximum pressure is shown in the figure, for convenience) had some visual similarities, but shows that the ellipsoids do not match the foot geometry perfectly. the plantar surface has many irregularities in its shape that is not captured by an ellipse. Because of these irregularities, the pressure error is expected to be larger than the the normal force and COP errors: while it is possible that different shapes could generate the same force profile, it is unlikely that they could generate the same pressure profile across the entire surface. Another reason this error could be inflated is due to measurement errors: it was noted from the experiments that the pressure mat used had a relatively long response time, resulting in some lag in the pressure profile and overall measuring slightly lower values than expected. 



Figure 5: Optimised volumetric contact model geometry

As a result, it is uncertain how much of the $33.9 \%$ RMS error in pressure was due to poor measurements from the pressure mat, and how much was due the inaccuracies of an ellipsoid volumetric contact model, especially since other similar studies did not measure experimental pressure or calculate the pressure error for comparison [16], [29].

Several methods could be used to improve the fit of volumetric contact to more complex geometry. Additional ellipsoids could be added to the model so that more of the surface is modelled (for example, based on Figure 6, a fourth ellipsoid at the lateral edge of the foot may improve the fit). The use of more complex, analytical shapes could also be explored, such as superellipsoids or superovoids [19]. These methods could improve the accuracy, but would also result in larger and likely more complex equations, decreasing the computational efficiency of the model. Exploring these other possibilities was left to a future study.

The toe angle shown in Figure 7 was determined entirely by the optimisation. Note that the toe angle may have an offset error: since the optimiser determined both the toe contact and the toe angle, it is possible that the toe contact was placed incorrectly and the toe angle was offset to account for the error. In this simulation, the toe angle is relatively constant between toe contact $(t=1.28 \mathrm{~s})$ and heel lift-off $(t=1.45 \mathrm{~s})$. After heel liftoff, the toe gradually extends (lifts up, relative to the foot) by 24 deg prior to toe lift-off $(t=1.88 \mathrm{~s})$. The angle prior to toe contact is irrelevant to this simulation since it has no impact on the results (since there are no toe contact forces). In a gait study in the literature, Zhu et al. noted that the toe angle stayed relatively constant until heel lift-off, after which it extended by $23 \mathrm{deg}$, and then quickly flexed by 13 deg prior to toe lift-off [33]. The optimised results presented here match these experimental results without the flexion immediately prior to toe lift-off. This may be because there is very little contact force during this period (since it is close to toe lift-off), so the extra flexion would have a negligible impact on the cost function.

The amount of motion correction used is also of interest, since this provides an estimate of the amount of error introduced by skin movement and the rigid body assumption. The 

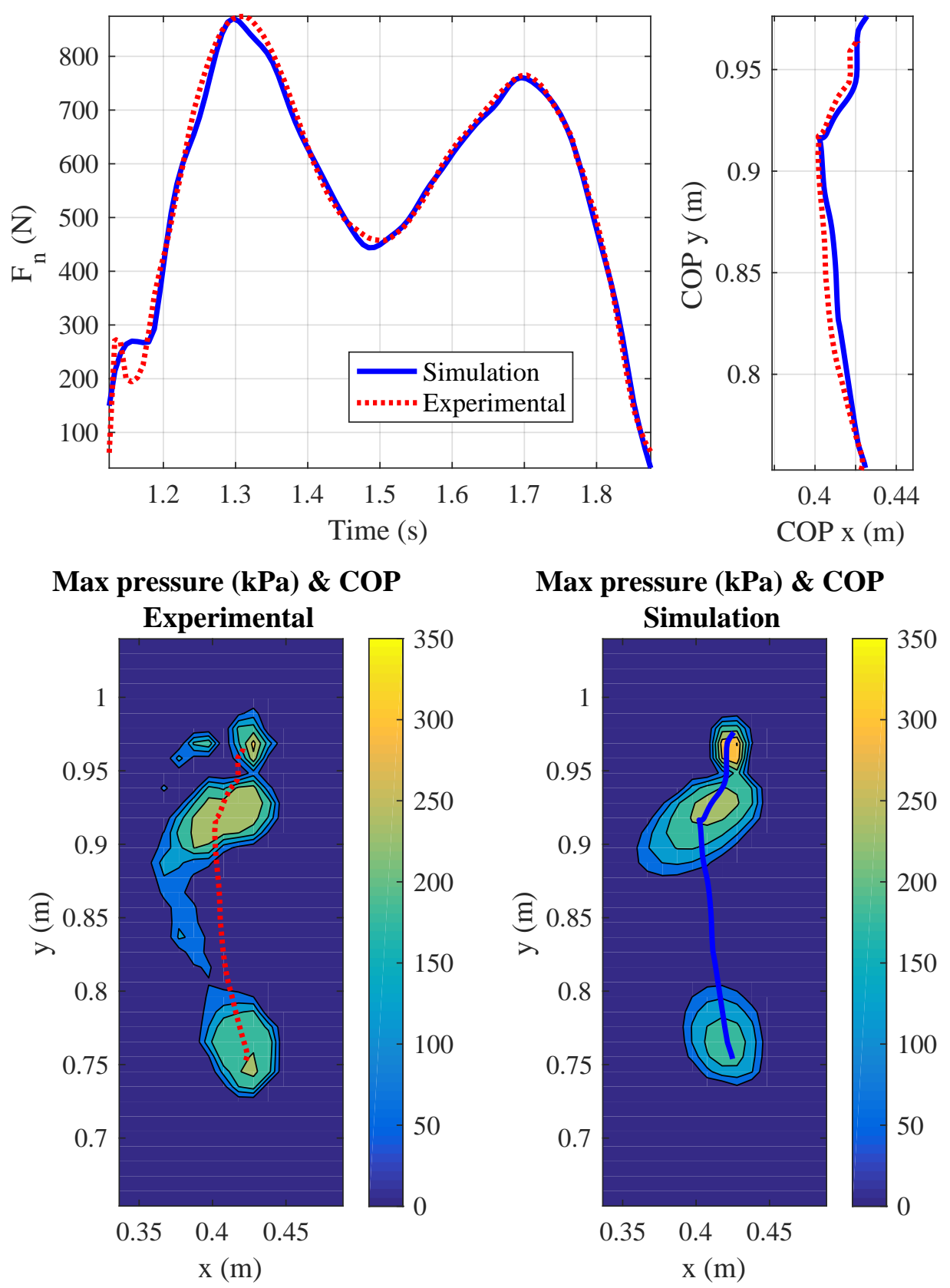

Figure 6: Simulated forces (top) and pressures (bottom) for optimal model 


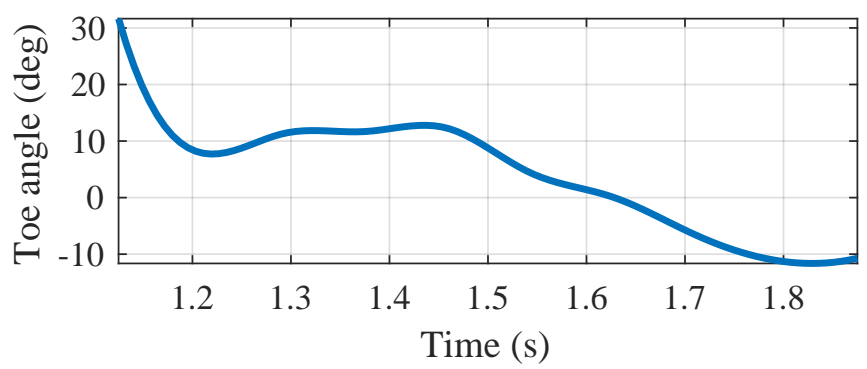

Figure 7: Optimised simulation toe actuation (relative to the foot). Negative angles indicate the toe lifting up (if the foot is flat on the ground).

RMS value of the motion correction applied is given in Table 5, where the displacement is relative to the the foot frame origin defined in Section 3.1.1 (the heel). The displacement correction is relatively low, under $2 \mathrm{~mm}$ RMS. The rotation angles are also relatively low, though the rotation correction about the $z$-axis is the largest (the reason for this was discussed in Section 3.2.1).

Table 5: RMS motion correction applied (relative to heel)

\begin{tabular}{|c|c|c|c|c|c|}
\hline \multicolumn{3}{|c|}{ Displacement (mm) } & \multicolumn{3}{|c|}{ Angle $^{\mathrm{a}}$ (deg) } \\
\hline $\mathrm{x}$ & $\mathrm{y}$ & $\mathrm{Z}$ & $\alpha$ & $\bar{\beta}$ & $\gamma$ \\
\hline 1.23 & 1.66 & 1.15 & 0.89 & 1.60 & 3.63 \\
\hline \multicolumn{6}{|c|}{$\begin{array}{l}\text { Rotation defir } \\
\text { Euler angles } \\
\text { the } x \text {-axis, } \beta \\
\text { axis }\end{array}$} \\
\hline
\end{tabular}

\subsection{Validation results}

The foot model parametrised in the calibration trial was used to simulate the other trials. The RMS errors for the normal force, COP, and pressure are given in Table 6. Just optimising the motion correction for the non-calibration trials took, on average, $82 \mathrm{~s}$.

Table 6: RMS error for foot-ground contact model

\begin{tabular}{c|c|c|c} 
Gait cadence (number of trials) & Normal force & COP & Pressure \\
\hline Calibration: 92 steps/minute (1) & $3.4 \%$ & $3.2 \%$ & $33.9 \%$ \\
92 steps/minute (3 ${ }^{\mathrm{a}}$ ) & $4.2 \%$ & $4.2 \%$ & $38.1 \%$ \\
112 steps/minute (5) & $4.9 \%$ & $6.6 \%$ & $44.1 \%$ \\
72 steps/minute (4 $\left.{ }^{\mathrm{a}}\right)$ & $4.4 \%$ & $5.0 \%$ & $40.0 \%$
\end{tabular}

a Some trials ignored due to unusual values or processing errors

As would be expected, the calibration trial had the lowest RMS errors, followed by gait trials at the same cadence. The higher cadence trials had the largest RMS values, though 
none of the trials had very different RMS errors from the calibration trial. The largest difference is between the calibration trial and the high cadence trial COP error, which was doubled. The pressure had the largest errors by almost an order of magnitude.

For comparison, Jackson et al. [16] developed a multiple point foot-ground contact model using similar methods for validation, except that validation was only done using gait at the same cadence. A comparison of the RMS errors for the simulations are given in Table 7.

Table 7: RMS error comparison with Jackson et al.

\begin{tabular}{c|c|c|c} 
& Normal force (N) & \multicolumn{2}{|c}{ COP $(\mathrm{mm})$} \\
& & $\mathrm{AP}^{\mathrm{a}}$ & $\mathrm{ML}^{\mathrm{b}}$ \\
\hline Volumetric & 28.8 & 8.0 & 2.4 \\
Jackson et al. [16] & 13 & 25 & 8
\end{tabular}

a Anterior-posterior direction

b Medial-lateral direction

The volumetric model presented here provided much better COP position matching compared to the model of Jackson et al., even for trials at different cadences, but the normal force did not match as closely. By heavily weighting the normal force term in the optimisation $\left(w_{\text {normal }}=500\right.$ and $\left.w_{C O P}=0.5\right)$, this model could obtain similar results to [16]; however, this reduced the fidelity of the COP, so these results were not explored. Some caution should be taken in comparing the results of these two models, since Jackson et al. used a different method to determine the motion correction and toe flexion and the subjects were of different weights.

\subsection{Results without Pressure in Optimisation}

Other studies in the literature, including Jackson et al. [16], did not use a pressure mat and the pressure data in determining foot parameters. To determine the effect of minimising the pressure errors in the optimisation, the same parameter optimisation was done without minimising pressure errors. For simplicity, only one calibration trial was done to compare the results with the calibration results in Section 4.1. The optimised model geometry is shown in Figure 8 and the results from the simulation are in Figure 9. Optimisation took about $10 \mathrm{~s}$.

Since the pressure distribution did not need to be calculated in the optimisation, the parameter optimisation took much less computational time: only $10 \mathrm{~s}$, compared to the previous $164 \mathrm{~s}$ average. The pressure distribution required calculating the pressure at a large number of points across the contact surface, rather than just the net force and contact location.

The optimised results matched the normal force and centre-of-pressure position more closely, because the optimisation was not also attempting to minimise the pressure distribution error. However, the resultant pressure distribution is a poor match. The rootmean-square error values for the optimisation with and without considering pressure are given in Table 8. 

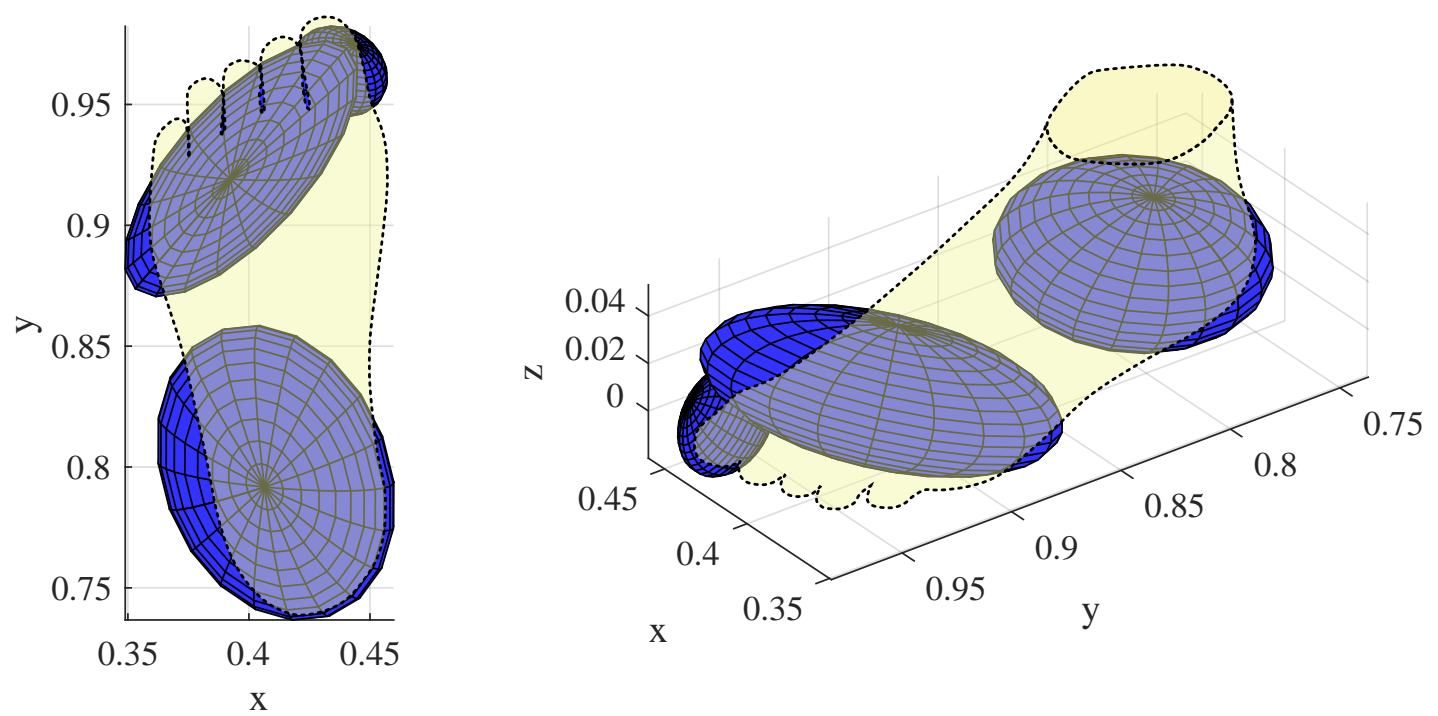

Figure 8: Optimised volumetric contact model geometry (optimisation without pressure distribution)

Table 8: RMS error for models with and without minimising contact pressure error

\begin{tabular}{c|c|c|c} 
Trial & Normal force & COP & Pressure \\
Calibration: 92 steps/minute & & & \\
\hline With pressure in optimisation & $3.4 \%$ & $3.2 \%$ & $33.9 \%$ \\
Without pressure in optimisation & $2.7 \%$ & $1.8 \%$ & $70.2 \%$
\end{tabular}



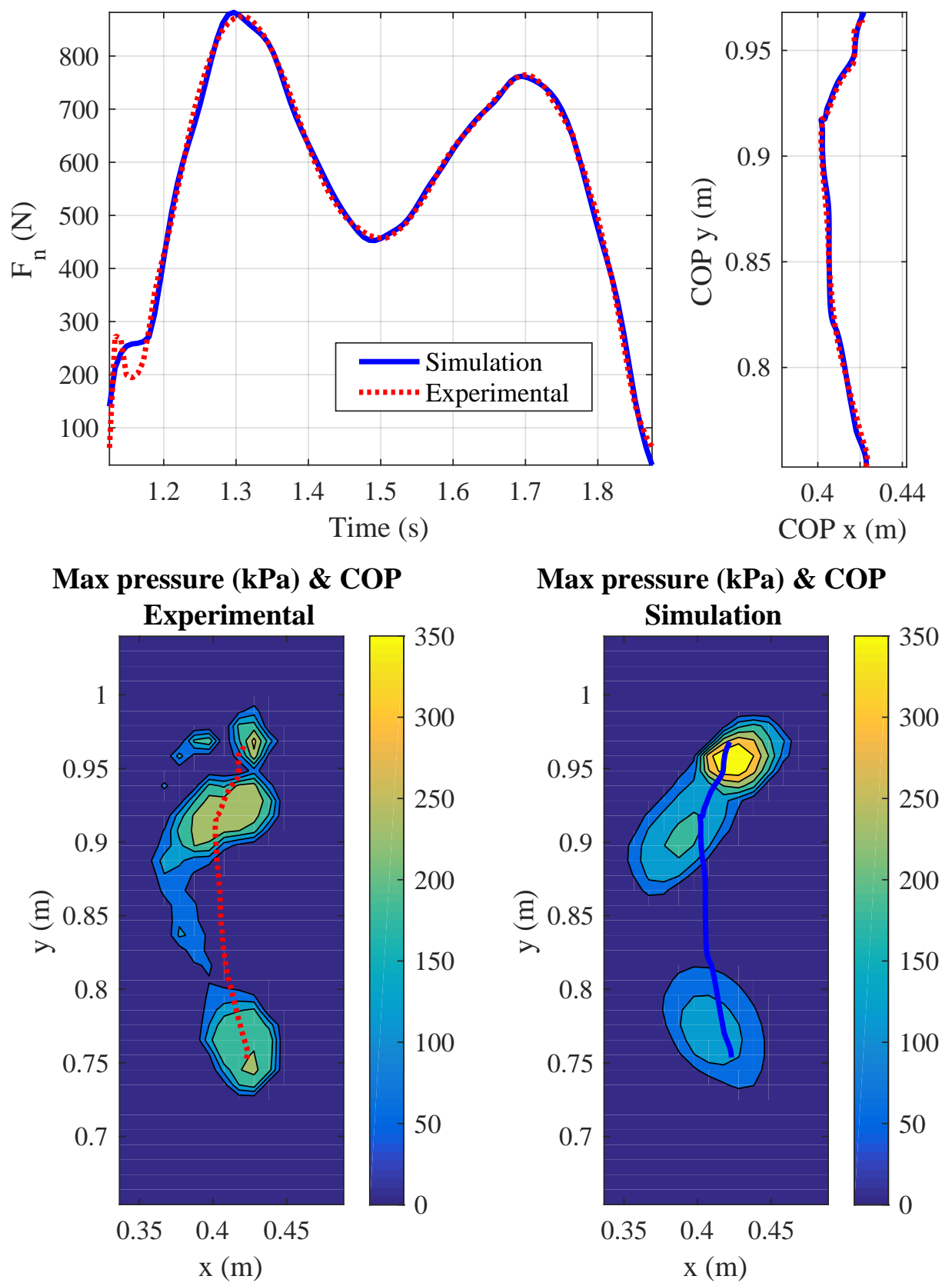

Figure 9: Simulated forces (top) and pressures (bottom) for optimisation not using pressure distribution 
The contact model (Figure 8) and pressure distribution (Figure 9) reveal that the 'toe' contact was placed between the toe and ball of the foot. This presumably happened to match the normal force and COP position more closely, but is an unrealistic representation of the foot geometry.

Also note that the toe rotation was solely determined by the optimisation, so it is uncertain if the "toe" movement here is physiological. The optimisation may have adapted the contact to represents parts of the foot surface other than the toe and converged to nonphysiological movement. This problem might be avoided by careful choice of optimisation bounds, but a better solution would be to design an experiment in which the foot movement and toe rotation can be calculated accurately.

Minimising pressure distribution error in the parameter optimisation increases computational time (by at least one order of magnitude) but helps avoid some non-physiological solutions, as presented in this section. It is possible that some of these errors may also have been avoided with a better designed experiment, but it is likely that minimising pressure errors helps converge to a more accurate model of the foot surface.

\section{$5 \quad$ Including Friction in the Model}

The same parametrisation was also done with a foot model that included friction. A continuous velocity-based friction model was used to approximate dynamic friction. However, simulation and parametrisation yielded inaccurate parameters and results. This was determined to be due to the methodology used (inverse dynamics simulation). Since these results were not useful for parameter identification, but are an interesting point of discussion, they are included in this section separate from the rest of the results.

\section{$5.1 \quad$ Model}

Friction was modelled using a modified form of the friction model originally designed for volumetric contact [22]. The original model is:

$$
\vec{F}_{t}=-F_{n} \mu \vec{v}_{c t}
$$

where $\vec{F}_{t}$ is the tangential friction force, $\mu$ the coefficient of friction, $F_{n}$ the magnitude of the normal force, and $\vec{v}_{c t}$ the tangential relative velocity at the centroid.

To obtain a friction model that more closely matched dynamic friction, a continuous velocity-based model was used [34]. Incorporating that friction equation into Equation (3) resulted in the following model:

$$
\vec{F}_{t}=-F_{n} \mu\left(\left|\vec{v}_{c t}\right|\right) \frac{\vec{v}_{c t}}{\left|\vec{v}_{c t}\right|}
$$

where

$$
\mu(v)=\mu_{d} \tanh \left(4 \frac{v}{v_{t}}\right)+\left(\mu_{s}-\mu_{d}\right) \frac{\frac{v}{v_{t}}}{\left(\frac{1}{4}\left(\frac{v}{v_{t}}\right)^{2}+\frac{3}{4}\right)^{2}}
$$


where $\mu_{d}$ is the dynamic coefficient of friction, $\mu_{s}$ the static coefficient of friction, and $v_{t}$ the transition velocity.

Similarly, the original model for spinning friction [22] was:

$$
\tau_{s}=-\mu \frac{F_{n}}{V} \mathbf{J} \omega
$$

where $\tau_{s}$ is spinning friction about the axis perpendicular to the plane, $\omega$ the rotational velocity about the same axis, and $\mathbf{J}$ is the weighted second moment of area (a value of interest in volumetric contact, defined in [22] and [34]). This was modified to be:

$$
\tau_{s}=-\frac{F_{n}}{V} \mu\left(\left|\omega_{c n}\right|\right) \mathbf{J} \frac{\omega_{c n}}{\left|\omega_{c n}\right|}
$$

where

$$
\mu(\omega)=\mu_{d} \tanh \left(4 \frac{\omega}{\omega_{t}}\right)+\left(\mu_{s}-\mu_{d}\right) \frac{\frac{\omega}{\omega_{t}}}{\left(\frac{1}{4}\left(\frac{\omega}{\omega_{t}}\right)^{2}+\frac{3}{4}\right)^{2}}
$$

where $\omega_{t}$ the transition angular velocity.

For simplicity in this initial model, the static friction term was ignored (i.e. $\mu_{s}=\mu_{d}$ ) and the friction transition parameters were set to arbitrary low values: $v_{t}=0.01 \mathrm{~m} / \mathrm{s}$ and $\omega_{t}=0.01 \mathrm{rad} / \mathrm{s}$. Thus, the only parameter for friction to be determined was the dynamic coefficient of friction.

\subsection{Results with Friction in Optimisation}

The weights for the optimisation function were all set to 1, except for the pressure term ( $\left.w_{\text {pressure }}\right)$ which was set to 0.1 due to the inaccuracies of the pressure measurements. The simulation results for the optimised solution are shown in Figure 10 and the RMS errors are shown in Table 9. Optimisation took $180 \mathrm{~s}$.

Table 9: RMS error for optimised model (including friction in optimisation)

\begin{tabular}{c|c|c|c|c|c} 
& Normal force & Friction force & Spinning friction & COP & Pressure \\
\hline With friction & $11.5 \%$ & $26.5 \%$ & $14.2 \%$ & $5.0 \%$ & $51.7 \%$ \\
Without friction & $3.4 \%$ & - & - & $3.2 \%$ & $33.9 \%$
\end{tabular}

With the addition of the friction terms, the simulated normal force was a poorer match to the experimental results (11.5\% RMS error instead of 3.4\%). The difference in normal force is especially noticeable around the minima at $t=1.45$ seconds in Figure 10 . The errors in the COP position and pressure profile also increased, though not to the same extent.

The friction forces did not match as well as the normal force did (26.5\% RMS error for tangential force, and $14.2 \%$ error for spinning friction). Also, the friction forces had regular jumps in magnitude or direction (Figure 10).

Upon further analysis, it was realised that the simulated friction force and normal force had a very similar trend. This is more visible if the net friction force $\left(\sqrt{F_{x}^{2}+F_{y}^{2}}\right)$ is shown with the normal force scaled by the coefficient of friction (Figure 11). 

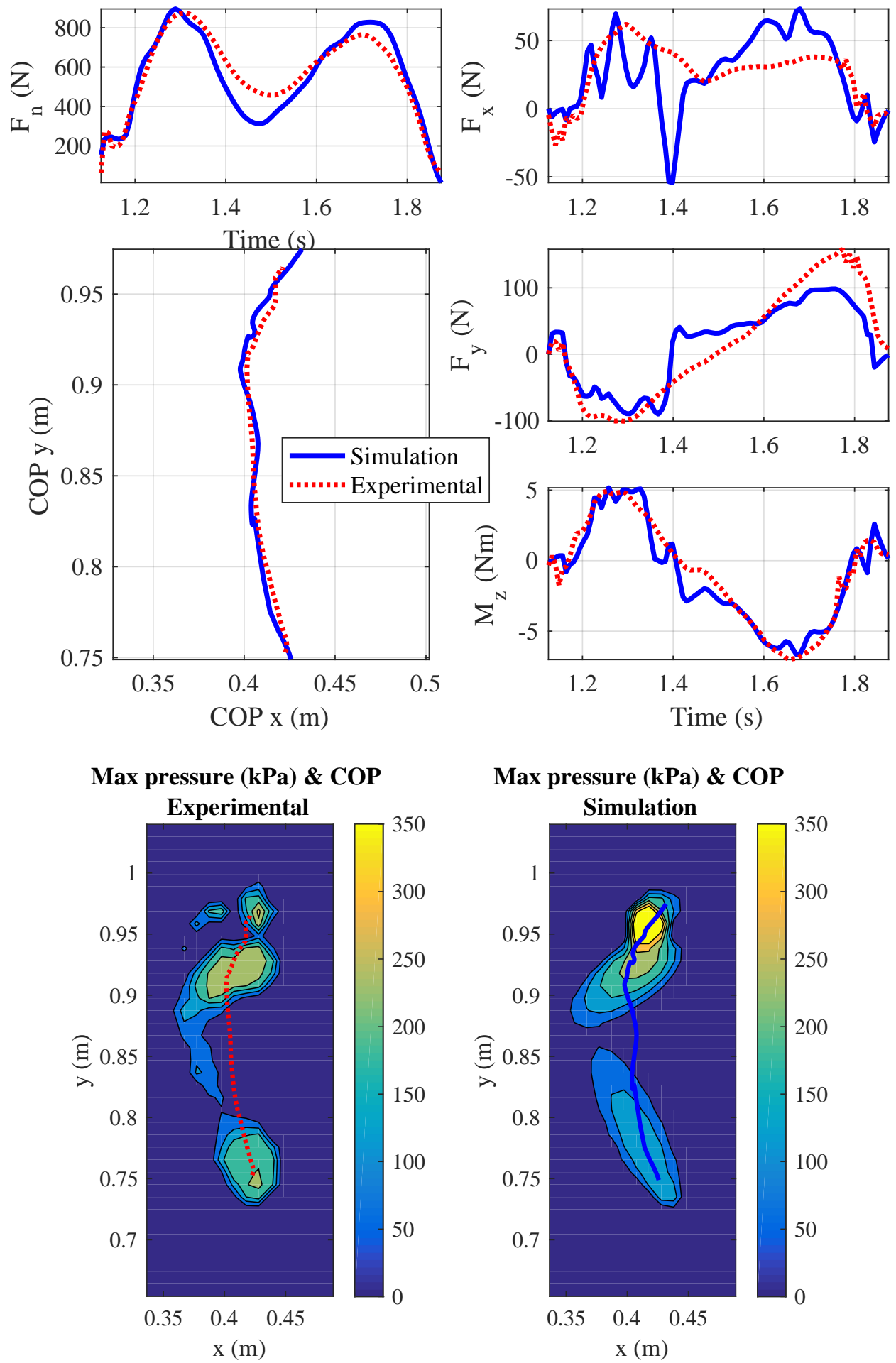

Figure 10: Simulated and experimental forces (top) and pressures (bottom) for optimised model (with friction). $F_{x}$ is medial-lateral force, $F_{y}$ is anterior-posterior force, and $M_{z}$ is the spinning moment. 


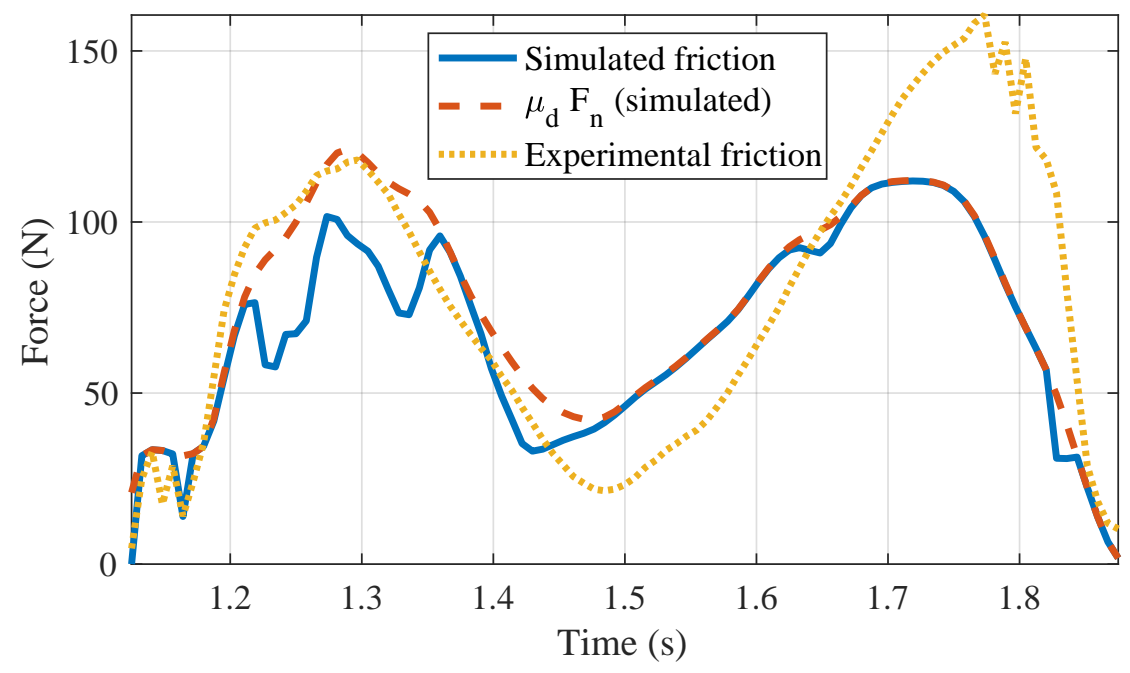

Figure 11: Simulated friction, maximum possible friction (based on normal force and $\mu_{d} F_{n}$ ), and experimental friction

In Figure 11, the simulated friction values generally follow the shape of the normal force (which dictates the maximum possible value for friction) more closely than the experimental friction values. It may also be noticed in Figure 10 that the simulated normal force had a lower-than-expected local minima around $t=1.47 \mathrm{~s}$; the optimisation appears to have converged to this so that the resulting friction forces match more closely. Another concern is that the simulated friction force was equal to $\mu_{d} F_{n}$ for most of the time, which would indicate that the foot was slipping; however, no slipping was noted during the experiment. Also worth noting is that the optimised friction coefficient, $\mu_{d}=0.14$, is much lower than expected ( $\mathrm{Li}$ and Wen measured a coefficient of friction of 0.65 for a bare foot on ceramic tiles $[35])$.

These issues can be explained as a limitation of using a velocity-based friction model in an inverse-dynamics simulation with little slipping. It is difficult for a velocity-based friction model to stay in the sticking regime in an inverse simulation. Velocity-based friction models assume that sticking occurs at very small relative velocities (in this model, less than $v_{t}=0.01 \mathrm{~m} / \mathrm{s}$ ) (Figure 12), despite the fact that true sticking would have a velocity of zero. In a forward dynamics simulation, which is driven by forces or moments acting on the bodies, the friction model results in a small level of error in the velocity. This small error is necessary to generate the sticking friction force, but the error does not exist in a real system and cannot be measured experimentally to drive an inverse simulation. In an inverse dynamics simulation, the measured motion of sticking surfaces should be zero, which would result in no friction. Any small errors in the velocity (from measurement noise or error) cause large changes in the friction force, driving the model to the sliding regime (due to the high sensitivity of friction force to velocity; see force-velocity curve in Figure 12).

A similar study in the literature is noted to have the same issue: Jackson et al. [16] used a similar method to parametrise a foot-ground contact model with a velocity-based 


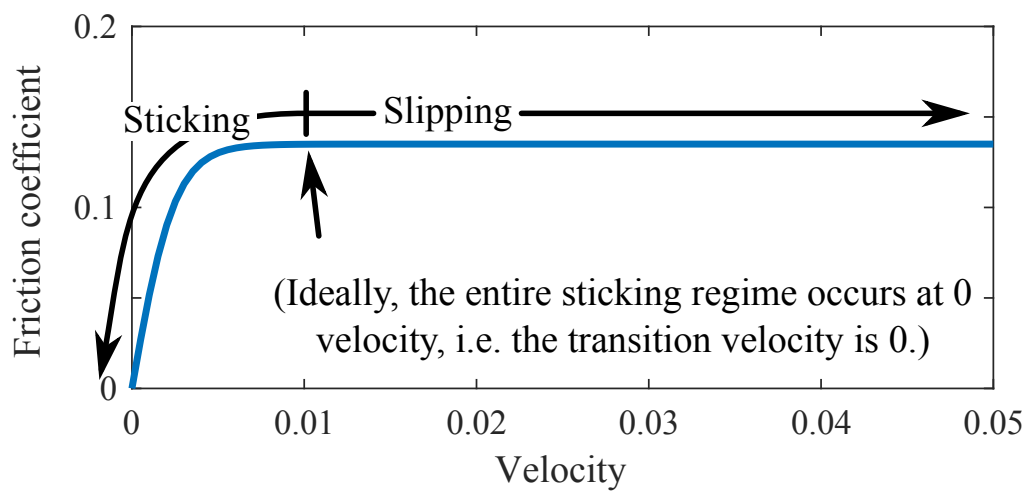

Figure 12: Velocity-based friction curve (only dynamic friction)

friction model, and obtained a static coefficient of friction of only 0.1 . This is particularly surprising since the subject was wearing sports shoes, which should have a higher coefficient of friction in order to provide good grip. In another study, Pàmies-Vilà et al. [29] noted that the model was unable to replicate the experimental friction well, hypothesising that the model may have been inadequate or the measurements of the foot movement may not have been accurate enough. In that study, a bristle friction model was used, which does not require small errors in velocity to model friction, but would still introduce large changes in friction forces due to any motion errors.

The coefficient of friction obtained in this optimisation was likely the average required coefficient of friction. The required coefficient of friction is calculated as the friction force divided by the normal force. Based on other studies, the maximum required coefficient of friction for walking is 0.15 to 0.30 [36], lending plausibility to the theory that the converged value of 0.14 is approximately the average required coefficient of friction for this study.

As a result, the coefficient of friction would be better identified by a separate experiment where dynamic friction occurs, such as dragging the foot across a force plate (such as in [35]) or in a forward dynamics simulation.

\section{Conclusions}

The developed foot-ground contact model was able to match experimental normal force and COP position within $5 \%$ for trials at the same or lower gait cadence. The COP position had higher levels of error (6.6\%) for a higher cadence. This model has better COP position accuracy, but poorer normal force accuracy than a recent model using a large number of point contacts [16]. The simulation was not able to match the experimental pressure as accurately: $33.9 \%$ RMS error for the calibration trial, and up to $44.1 \%$ RMS error for trials at other cadences. Some of this error may be due to measurement error with the pressure mat, and it is difficult to compare to other studies in the literature since pressure is not generally measured.

The model parameter optimisation was sensitive to the initial guess and a good guess was required to converge to the given results. It is uncertain if the provided results are the global minimum, but the developed model was able to closely match the experimental 
results. As noted by [16], there may be more than one combination of parameters with good prediction results.

Inverse dynamics simulation and parameter optimisation proved to be an inaccurate method for determining friction parameters and validating the friction model. Unusually low coefficients of friction were found and the optimisation converged to unusual results (such as adjusting the normal force profile to better match the friction force profile). A different method for determining the coefficient of friction is recommended (such as dragging the foot on a force plate), and the friction model must be validated in a forward dynamics simulation.

The rigid body simplification for the foot introduced inaccuracies when determining foot motion from the gait experiment. To continue using this simplification, the optimisation was allowed to apply some motion correction to the experimental data. Using multiple rigid bodies to represent the foot segment and using additional markers (or stick markers) to measure the foot motion could improve the accuracy of the motion determined from the experiment. This might reduce the need for the motion correction introduced in this study.

Despite the inaccuracies of the pressure mat, minimising the pressure errors as part of the parameter optimisation appears to result in more realistic model geometry. This comes at an increase in computational cost in the parameter optimisation. This increased computational cost is only present during parametrisation; the resulting models are equivalent in terms of computational cost. The increased optimisation time (in this study) came with the benefit of converging to a more physiological solution. In future work, results from this study may be improved with a more accurate pressure mat with a faster response time.

The model parameters given here are based on a single subject and a single activity

and may not be generalisable or statistically significant. Further studies are necessary to confirm this model can be used for other subjects with appropriate changes in parameters. Using several activities to parametrise the model (walking, jumping, and running, etc.) may help improve the model accuracy for a generalised movement. Only using a single subject may be beneficial, however, since subject-specific contact models tend to improve accuracy.

In future studies, to improve the volumetric contact model, different numbers of analytical shapes or more complex shapes, such as superellipsoids or superovoids, could be considered. This could improve the accuracy of the contact model by more closely approximating the actual shape of the plantar surface at some computational cost. It would also be interesting to compare the contact surface geometry determined through parameter estimation to geometry measured directly from the surface.

\section{Acknowledgements}

This research was funded by the Natural Sciences and Engineering Research Council of Canada and the Canada Research Chairs program.

Special thanks to Dr. Stacey Acker and David Kingston for the use of their lab space and assistance running the gait experiments. 


\section{References}

[1] F. C. Anderson and M. G. Pandy, "Dynamic optimization of human walking," ASME Journal of Biomechanical Engineering, vol. 123, no. 5, pp. 381-390, 2001.

[2] M. Peasgood, E. Kubica, and J. McPhee, "Stabilization of a dynamic walking gait simulation," ASME Journal of Computational and Nonlinear Dynamics, vol. 2, no. 1, pp. 65-72, 2007.

[3] C. R. Smith, M. F. Vignos, R. L. Lenhart, J. Kaiser, and D. G. Thelen, "The influence of component alignment and ligament properties on tibiofemoral contact forces in total knee replacement," ASME Journal of Biomechanical Engineering, vol. 138, no. 2, 021017:1-10, 2016.

[4] B. J. Fregly, J. A. Reinbolt, K. L. Rooney, K. H. Mitchell, and T. L. Chmielewski, "Design of patient-specific gait modifications for knee osteoarthritis rehabilitation," IEEE Transactions on Biomedical Engineering, vol. 54, no. 9, pp. 1687-1695, 2007.

[5] S. J. Allen, M. A. King, and M. R. Yeadon, "Optimisation of phase ratio in the triple jump using computer simulation," Human Movement Science, vol. 46, pp. 167-176, 2016.

[6] J. P. Halloran, M. Ackermann, A. Erdemir, and A. J. van den Bogert, "Concurrent musculoskeletal dynamics and finite element analysis predicts altered gait patterns to reduce foot tissue loading," Journal of Biomechanics, vol. 43, no. 14, pp. 2810-2815, 2010 .

[7] P. Saraswat, M. S. Andersen, and B. A. MacWilliams, "A musculoskeletal foot model for clinical gait analysis," Journal of Biomechanics, vol. 43, pp. 1645-1652, 2010.

[8] R. Fluit, M. S. Andersen, S. Kolk, N. Verdonschot, and H. F. J. M. Koopman, "Prediction of ground reaction forces and moments during various activities of daily living," Journal of Biomechanics, vol. 47, no. 10, pp. 2321-2329, 2014.

[9] S. Skals, M. K. Jung, M. Damsgaard, and M. S. Andersen, "Prediction of ground reaction forces and moments during sports-related movements," Multibody System Dynamics, pp. 1-21, 2016.

[10] J. M. Font-Llagunes, A. Barjau, R. Pàmies-Vilà, and J. Kövecses, "Dynamic analysis of impact in swing-through crutch gait using impulsive and continuous contact models," Multibody System Dynamics, vol. 28, no. 3, pp. 257-282, 2012.

[11] J. Ros, J. M. Font-Llagunes, A. Plaza, and J. Kövecses, "Dynamic considerations of heel-strike impact in human gait," Multibody System Dynamics, vol. 35, no. 3, pp. 215-232, 2015.

[12] A. Pérez-González, C. Fenollosa-Esteve, J. L. Sancho-Bru, F. T. Sánchez-Marín, M. Vergara, and P. J. Rodríguez-Cervantes, "A modified elastic foundation contact model for application in 3D models of the prosthetic knee," Medical Engineering and Physics, vol. 30, no. 3, pp. 387-398, 2008. 
[13] P. Flores, M. Machado, M. T. Silva, and J. M. Martins, "On the continuous contact force models for soft materials in multibody dynamics," Multibody System Dynamics, vol. 25, no. 3, pp. 357-375, 2011.

[14] A. Mahboobin, R. Cham, and S. J. Piazza, "The impact of a systematic reduction in shoe-floor friction on heel contact walking kinematics-a gait simulation approach," Journal of Biomechanics, vol. 43, pp. 1532-1539, 2010.

[15] D. S. Lopes, R. R. Neptune, J. A. Ambrósio, and M. T. Silva, "A superellipsoid-plane model for simulating foot-ground contact during human gait," Computer Methods in Biomechanics and Biomedical Engineering, pp. 1-10, 2015.

[16] J. N. Jackson, C. J. Hass, and B. J. Fregly, "Development of a subject-specific footground contact model for walking," ASME Journal of Biomechanical Engineering, vol. 138, no. 9, 091002:1-12, 2016.

[17] P. Moreira, M. Silva, and P. Flores, "Ground foot interaction in human gait: Modelling and simulation," in Proceedings of rth EUROMECH Solid Mechanics Conference, Lisbon, Portugal, \%-11 September, 2009.

[18] M. Millard and A. Kecskeméthy, "A 3D foot-ground model using disk contacts," in The 3rd Joint International Conference on Multibody System Dynamics \& The 7th Asian Conference on Multibody Dynamics, Busan, Korea, 2014.

[19] A. A. Gonçalves, A. Bernardino, J. Jorge, and D. S. Lopes, "A benchmark study on accuracy-controlled distance calculation between superellipsoid and superovoid contact geometries," Mechanism and Machine Theory, vol. 115, pp. 77-96, 2017.

[20] Y. Gonthier, J. McPhee, C. Lange, and J.-C. Piedbœuf, "A contact modeling method based on volumetric properties," in ASME IDETC, Long Beach, California, USA, 2005, pp. 477-486.

[21] M. Boos and J. McPhee, "Volumetric modeling and experimental validation of normal contact dynamic forces," ASME Journal of Computational and Nonlinear Dynamics, vol. 8, no. 2, 021006:1-8, 2013.

[22] Y. Gonthier, "Contact dynamics modelling for robotic task simulation," PhD Thesis, University of Waterloo, 2007.

[23] M. Millard, J. McPhee, and E. Kubica, "Multi-step forward dynamic gait simulation," in Multibody Dynamics: Computational Methods and Applications, C. L. Bottasso, Ed., Dordrecht: Springer Netherlands, 2009, pp. 25-43.

[24] M. Sharif Shourijeh and J. McPhee, "Forward dynamic optimization of human gait simulations: A global parameterization approach," ASME Journal of Computational and Nonlinear Dynamics, vol. 9, no. 3, 031018:1-11, 2014.

[25] M. S. Shourijeh and J. McPhee, "Foot-ground contact modeling within human gait simulations: From Kelvin-Voigt to hyper-volumetric models," Multibody System Dynamics, vol. 35, no. 4, pp. 393-407, 2015. 
[26] F. Mouzo, U. Lugrís, R. Pàmies-Vilà, J. M. Font-Llagunes, and J. Cuadrado, "Underactuated approach for the control-based forward dynamic analysis of acquired gait motions," in ECCOMAS Thematic Conference on Multibody Dynamics, Barcelona, Catalonia, Spain, 2015.

[27] P. Brown, "Contact modelling for forward dynamics of human motion," Master's thesis, University of Waterloo, 2017.

[28] P. Brown and J. McPhee, "Volumetric contact model of ellipsoid-plane geometries," in ECCOMAS Thematic Conference on Multibody Dynamics, 2017.

[29] R. Pàmies-Vilà, J. M. Font-Llagunes, U. Lugrís, and J. Cuadrado, "Parameter identification method for a three-dimensional foot-ground contact model," Mechanism and Machine Theory, vol. 75, pp. 107-116, 2014.

[30] P. Gerus, M. Sartori, T. F. Besier, B. J. Fregly, S. L. Delp, S. A. Banks, M. G. Pandy, D. D. D'Lima, and D. G. Lloyd, "Subject-specific knee joint geometry improves predictions of medial tibiofemoral contact forces," Journal of Biomechanics, vol. 46, no. 16, pp. 2778-2786, 2013.

[31] M. Wesseling, F. De Groote, L. Bosmans, W. Bartels, C. Meyer, K. Desloovere, and I. Jonkers, "Subject-specific geometrical detail rather than cost function formulation affects hip loading calculation," Computer Methods in Biomechanics and Biomedical Engineering, vol. 19, no. 14, pp. 1475-1488, 2016.

[32] I. Hannah, Z. Sawacha, A. Guiotto, and C. Mazzà, "Relationship between sagittal plane kinematics, foot morphology and vertical forces applied to three regions of the foot," International Biomechanics, vol. 3, no. 1, pp. 50-56, 2016.

[33] J. Zhu, Q. Wang, and L. Wang, "On the design of a powered transtibial prosthesis with stiffness adaptable ankle and toe joints," IEEE Transactions on Industrial Electronics, vol. 61, no. 9, pp. 4797-4807, 2014.

[34] P. Brown and J. McPhee, "A continuous velocity-based friction model for dynamics and control with physically meaningful parameters," ASME Journal of Computational and Nonlinear Dynamics, vol. 11, no. 5, 054502:1-6, 2016.

[35] K. W. Li and H. C. Wen, "Friction between foot and floor under barefoot conditions: A pilot study," IEEE International Conference on Industrial Engineering and Engineering Management, pp. 1651-1655, 2014.

[36] R. Grönqvist, J. Abeysekera, G. Gard, S. M. Hsiang, T. B. Leamon, D. J. Newman, K. Gielo-Perczak, T. E. Lockhart, and C. Y. Pai, "Human-centred approaches in slipperiness measurement," Ergonomics, vol. 44, no. 13, pp. 1167-1199, 2001. 